\title{
ARTICLE
}

Cancer Metabolism

\section{Inactivation of 3-hydroxybutyrate dehydrogenase type 2 promotes proliferation and metastasis of nasopharyngeal carcinoma by iron retention}

\author{
Bo $\mathrm{Li}^{1}$, Zhipeng Liao ${ }^{2}$, Yingxi Mo ${ }^{3}$, Weilin Zhao ${ }^{2}$, Xiaohui Zhou ${ }^{4}$, Xiling Xiao ${ }^{2}$, Wanmeng Cui ${ }^{5}$, Guofei Feng ${ }^{2}$, Suhua Zhong ${ }^{2}$,
} Yushan Liang ${ }^{2}$, Chunping $\mathrm{Du}^{6}$, Guangwu Huang ${ }^{2}$, Ping $\mathrm{Li}^{4}$, Xue Xiao ${ }^{2}$, Xiaoying Zhou ${ }^{6}$, Rensheng Wang ${ }^{1}$ and $\mathrm{Zhe} \mathrm{Zhang}^{2}$

BACKGROUND: 3-Hydroxybutyrate dehydrogenase type $2(\mathrm{BDH} 2)$ is known to catalyse a rate-limiting step in the biogenesis of the mammalian siderophore and regulate intracellular iron metabolism. Here we aim to explore the expression and possible function of $\mathrm{BDH} 2$ in nasopharyngeal carcinoma (NPC).

METHODS: The transcription and protein expression of BDH2 in NPC were determined by both real-time RT-PCR and immunohistochemistry staining assays. Cell proliferation, migration and invasion were evaluated by MTT assay, wound-healing assay and Transwell assay, respectively. The profile of genes regulated by restoring BDH2 expression in NPC cells was analysed by cDNA microarray. The level of iron in NPC cells was detected by iron colorimetric assay.

RESULTS: The expression of $\mathrm{BDH} 2$ was significantly downregulated in NPC. Ectopic expression of BDH2 inhibited NPC cell proliferation and colony formation. Meanwhile, $\mathrm{BDH} 2$ suppressed the migration and invasion of NPC cells by reversing the epithelial-mesenchymal transition (EMT). In addition, a higher level of $\mathrm{BDH} 2$ decreased the growth and metastasis of NPC cells via reducing intracellular iron level.

CONCLUSIONS: Our findings suggest that BDH2 may be a candidate tumour-suppressor gene in NPC. Decreasing intracellular iron could be an effective therapeutic approach for NPC.

British Journal of Cancer (2020) 122:102-110; https://doi.org/10.1038/s41416-019-0638-8

\section{BACKGROUND}

Nasopharyngeal carcinoma (NPC) is a malignant tumour with the distinct geographical, racial and ethnic distribution. ${ }^{1}$ For most western countries, NPC is an uncommon malignancy with an incidence of $<1 / 100,000$ people. ${ }^{2}$ However, this cancer is prevalent in southern China, where it is the third most common malignancy among males, with an incidence of $15-30 / 100,000$ people. ${ }^{3}$ Three major aetiological factors are involved in the carcinogenesis of NPC, including genetic susceptibility, environmental factors and Epstein-Barr virus (EBV) infection. ${ }^{1,3}$ Currently, the major therapeutic options of NPC include radiotherapy, chemotherapy and chemoradiotherapy. Despite significant progress in intensitymodulated radiation therapy and chemoradiotherapy, therapy for NPC still remains a great challenge because of radio- and chemo-resistance, disease relapse and distant metastasis. Therefore, we need novel and effective therapeutic strategies for NPC. ${ }^{4}$

Iron-generated reactive oxygen/nitrogen species and aldehydes lead to genetic alteration, and elevated iron levels might increase the risk of cancer. ${ }^{5}$ Excess iron levels have been found carcinogenic in many animal models. ${ }^{6}$ Iron acts as a cofactor for many enzymes including ribonucleotide reductase, an enzyme involved in NDA synthesis. To sustain proliferation, cancer cells need to increase the iron assimilation and consequently modulate the expression of proteins involved in iron uptake. Decreased expression of the iron exporter ferroportin leading to iron retention was found to be associated with poor prognosis in breast cancer. ${ }^{7}$

3-Hydroxybutyrate dehydrogenase type $2(\mathrm{BDH} 2)$, also called DHRS6 and located on the human chromosome 4q24, encodes a member of the short-chain dehydrogenase/reductase family. ${ }^{8}$ As a key enzyme in ketogenesis, $\mathrm{BDH} 2$ mediates the first step of ketone body degradation from 3-hydroxybutyrate to acetoacetate in the liver. ${ }^{9}$ In tissues other than the liver, $\mathrm{BDH} 2$ has a physiological role in the utilisation of cytosolic ketone bodies, as a secondary system for energy supply in starvation or to generate precursors for lipid and sterol synthesis. ${ }^{8} \mathrm{BDH} 2$ also manipulates intracellular iron homoeostasis by containing an iron-responsive element and mediating cellular iron tracking by catalysing the synthesis of the mammalian siderophore that binds labile iron. ${ }^{10}$

The roles of $\mathrm{BDH} 2$ differ in several human malignancies. $\mathrm{BDH} 2$ mRNA expression was higher in the bone marrow of patients with

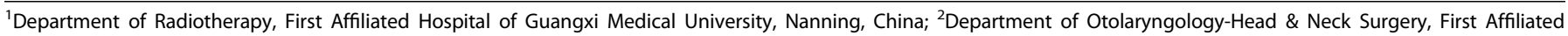

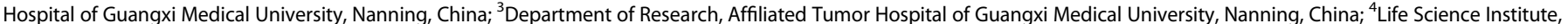

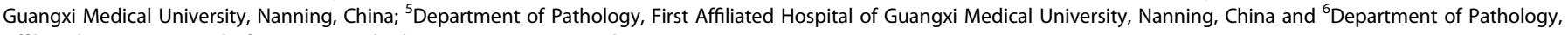
Affiliated Tumor Hospital of Guangxi Medical University, Nanning, China

Correspondence: Rensheng Wang (13807806008@163.com) or Zhe Zhang (zhangzhe@gxmu.edu.cn)

These authors contributed equally: Bo Li, Zhipeng Liao

Received: 27 April 2019 Revised: 5 September 2019 Accepted: 28 October 2019

Published online: 10 December 2019 


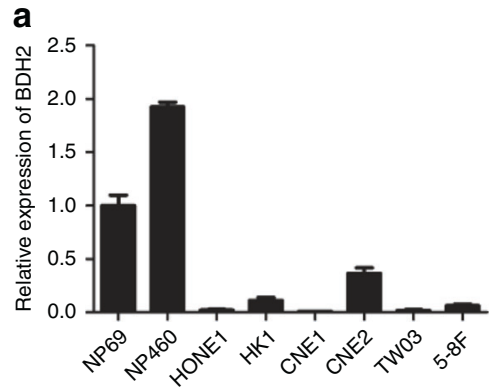

b
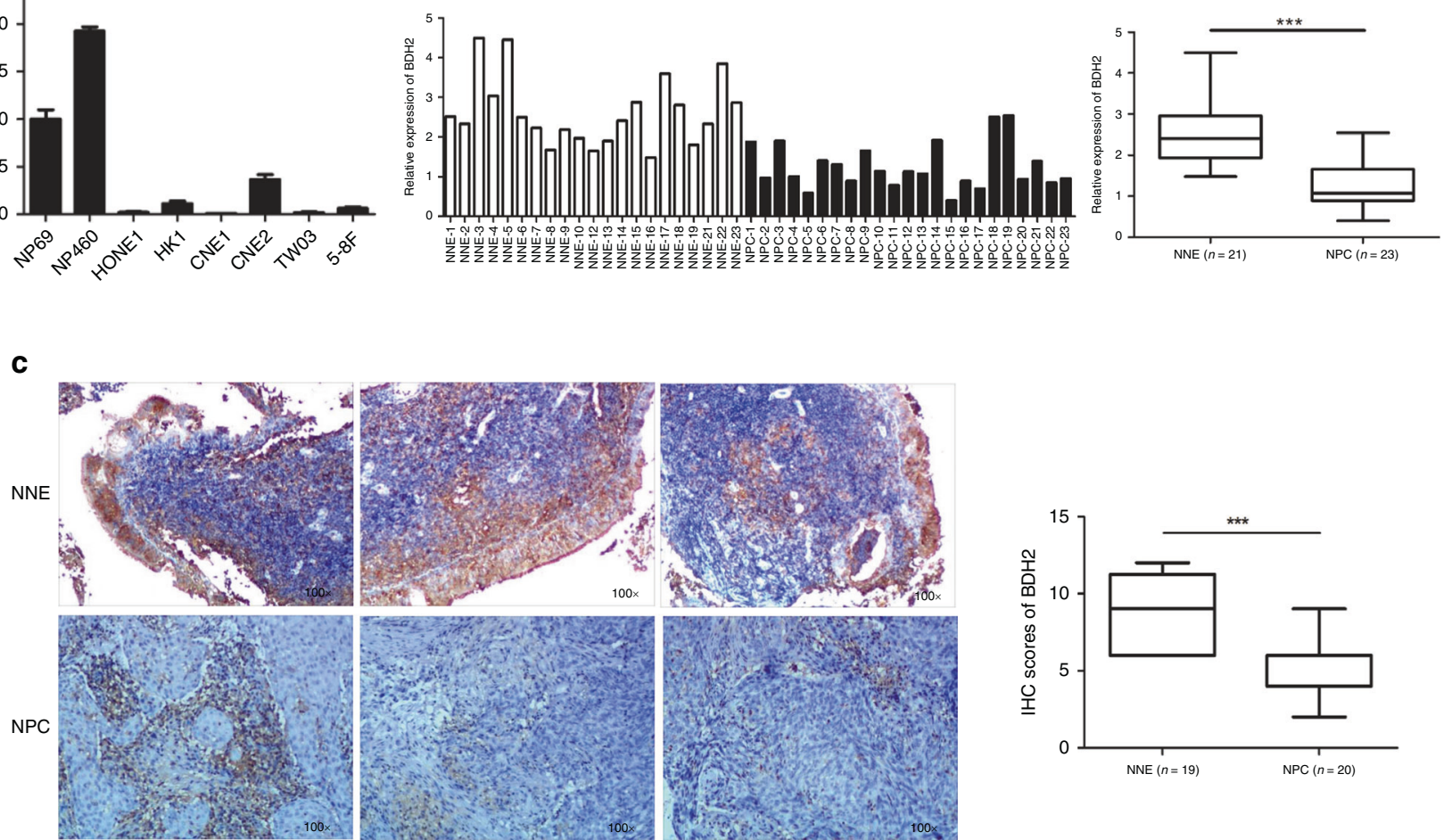

Fig. 1 Downregulated mRNA and protein level of BDH2 in NPC. a Relative BDH2 transcriptional level was determined by real-time RT-PCR in six NPC cells and two noncancerous nasopharyngeal epithelial (NNE; NP69, NP460) cell lines by using the $2^{-\Delta C t}$ method and normalised to GAPDH. b Real-time RT-PCR analysis of relative BDH2 mRNA expression in normal $(n=21)$ and tumour $(n=23)$ tissues. c Representative images of immunostaining for BDH2 expression in three NNE and three NPC samples (magnification $\times 100$ ). Horizontal line indicates the median, box edges are $25-75$ percentiles and whiskers are 10 and 90 percentiles. ${ }^{* * *} p<0.001$

acute myeloid leukaemia (AML). Overall survival was shorter in AML patients with higher $\mathrm{BDH} 2$ expression, and the response to intensive induction chemotherapy was lower. $\mathrm{BDH} 2$ works as an antiapoptotic factor, mediated by survivin via a caspase-3-independent pathway. ${ }^{11}$ Besides, $\mathrm{BDH} 2$ was found upregulated and correlated with tumour location or TNM stage in oesophageal cancer tissue. Knockdown of $\mathrm{BDH} 2$ induced cell apoptosis via a caspase-3dependent apoptosis pathway. ${ }^{12}$ On the other hand, the expression of $\mathrm{BDH} 2$ was decreased in hypoxic glioma cells. ${ }^{13}$ However, the expression and function of $\mathrm{BDH} 2$ remain unclear in NPC.

Here, we explored the expression of $\mathrm{BDH} 2$ in NPC, addressed its effect on malignant behaviour and possible molecular mechanisms involved.

\section{METHODS}

Cell lines and culture conditions

The immortalised human nasopharyngeal epithelial cell lines NP69 and NP460 were maintained in keratinocyte/serum-free medium (Invitrogen, Carlsbad, CA, USA). ${ }^{14,15}$ The NPC cell lines C666-1, HONE1, CNE1, HK1, 5-8 F, 6-10B and TW03 were maintained in high glucose DMEM (Invitrogen) supplemented with $10 \%$ foetal bovine serum (Invitrogen), $100 \mathrm{U} / \mathrm{mL}$ penicillin and $100 \mu \mathrm{g} / \mathrm{mL}$ streptomycin. Cells were incubated at $37^{\circ} \mathrm{C}$ in a humidified chamber containing $5 \% \mathrm{CO}_{2}{ }^{16-19}$

Primary NPC tumour biopsies were obtained from 43 newly diagnosed and untreated cases from donors, with informed consent, in the Department of Otolaryngology, Head and Neck Surgery, First Affiliated Hospital of Guangxi Medical University (Nanning, China). The diagnoses were established by experienced pathologists according to the World Health Organization (WHO) classification. Forty normal nasopharyngeal epithelium (NNE) tissue samples were collected as controls. In total, 21 NNE and 23 NPC biopsies were used for RNA extraction, and 19 NNE and 20 NPC biopsies were fixed in $10 \%$ paraformaldehyde embedded in paraffin.

Antibodies, plasmids and transfection

We used the following antibodies: BDH2 (1:1000, HPA004428, Sigma-Aldrich, St. Louis, MO, USA), $\beta$-catenin (1:1000, sc-376841, Santa Cruz Biotechnology, Santa Cruz, CA, USA), E-cadherin (1:1000, \#3195P) and vimentin (VIM; 1:1000, \#5741P, both Cell Signaling Technology, Beverly, USA), secreted protein acidic and cysteine rich (SPARC; 1:1000, \#66426-1, SANYING, Wuhan, China) and GAPDH (1:10000, \#5174P, Cell Signaling Technology). The secondary antibody 680RD goat anti-mouse and IRDye $800 \mathrm{CW}$ goat antirabbit antibodies were from LI-COR Biosciences (Lincoln, NE, USA).

$\mathrm{BDH} 2$ plasmid was purchased from Origene (Rockville, MD, USA); the open-reading frame of $\mathrm{BDH} 2$ was subcloned into the pCMV6Entry vector. The protocol of transfection has been described. ${ }^{20}$

RNA extraction, CDNA synthesis and real-time PCR

The RNA extraction, cDNA synthesis and real-time PCR were performed as previously described. ${ }^{20}$ The primer sequences were as follows: BDH2-F 5'-GCTTCCAGCGTCAAAGGAGT1-3', BDH2-R 5'CAGTTGCGAATCTTCCCGTC-3', GAPDH-F 5'-GCTCAGACACCATGGG GAAG-3', GAPDH-R 5'-TGTAGTTGAGGTCAATGAAGGGG-3', epithelial cell adhesion molecule (EPCAM)-F 5'-CTGGCCGTAAACTGC TTTGT-3', EPCAM-R 5'-AGCCCATCATTGTTCTGGAG-3', SPARC-F 5'GTGCAGAGGAAACCGAAGAG-3', SPARC-R 5'-TCATTGCTGCACACC TTCTC-3', cadherin 1 (CDH1)-F 5'-GTCAGGTGCCTGAGAACGAG-3', CDH1-R 5'-GCCATCGTTGTTCACTGGAT-3', VIM-F 5'- GAACTITGCCG TTGAAGCTG-3', VIM-R 5'-TCTCAATGTCAAGGGCCATC-3', matrix metalloproteinase 2 (MMP2)-F 5'-GCCTCCTCCTGACATTGACCT-3' and MMP2-R 5'-AACACAGCCTTCTCCTCCTG-3'. 


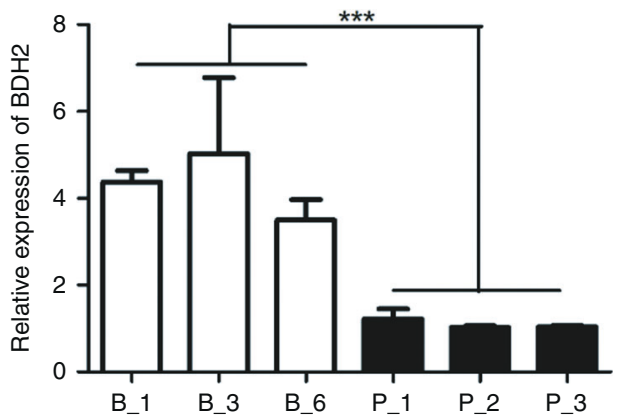

C

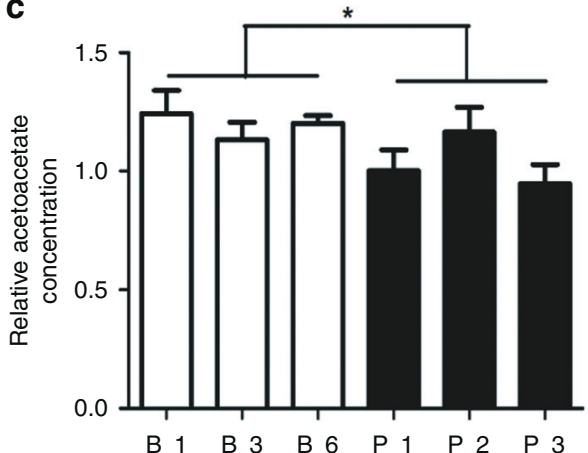

e

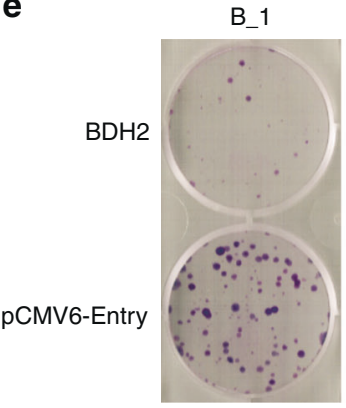

P_1
B_3

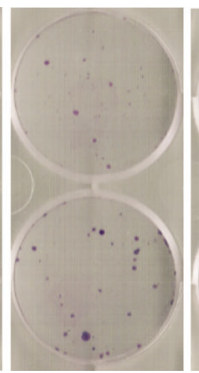

P_2
B_6

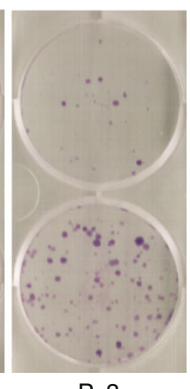

b

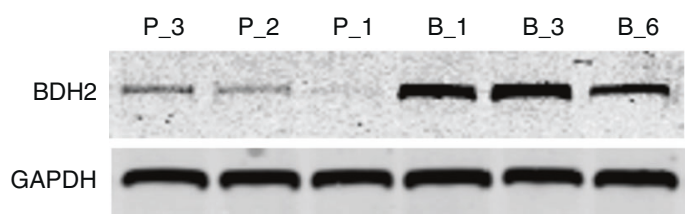

d
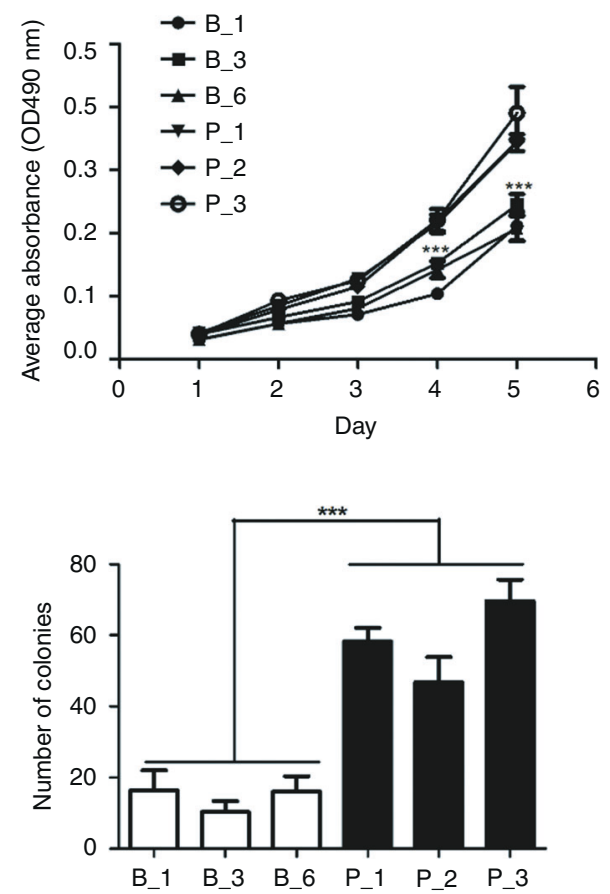

Fig. 2 Ectopic expression of $\mathrm{BDH} 2$ inhibits the proliferation and colony formation of NPC cells. $\mathbf{a}, \mathbf{b}$ The expression of BDH2 in $5-8 \mathrm{~F}$ cells stably transfected with $\mathrm{BDH}$ 2-PCMV6-Entry $\left(\mathrm{B}_{-}\right)$and control vector $\left(\mathrm{P}_{-}\right)$, respectively, confirmed by both real-time RT-PCR and western-blot analysis. c Acetoacetate detection assay in $\mathrm{BDH} 2-5-8 \mathrm{~F}$ and $\mathrm{pCMV6}-5-8 \mathrm{~F}$ cells. d Growth curve of three sub-colonies of $\mathrm{BDH} 2-5-8 \mathrm{~F}$ and $\mathrm{pCMV6}-5-8 \mathrm{~F}$ cells determined by MTT assay. e Colony formation of $\mathrm{BDH} 2-5-8 \mathrm{~F}$ and $\mathrm{pCMV} 6-5-8 \mathrm{~F}$ cells. Data are mean \pm SD. ${ }^{*} P<0.05,{ }^{* * *} P<0.001$

Immunohistochemistry staining

Tissues were cut into 3 - $\mu$ m-thick sections and incubated for $1 \mathrm{~h}$ with $3 \%$ hydrogen peroxide to eliminate endogenous peroxidase activity after deparaffinisation and rehydration. After antigen retrieval, sections were incubated with $\mathrm{BDH} 2$ antibody (1:1000, HPA004428, Sigma, St. Louis, MO, USA) at $4{ }^{\circ} \mathrm{C}$ overnight, then secondary antibody (ZB-2305, ZSGB-BIO, Beijing) for $1 \mathrm{~h}$ at room temperature. A 3,3'-diaminobenzidine (DAB) reagent (ZLI-9018, ZSGB-BIO, Beijing) was used for peroxidase reaction, and haematoxylin for counterstaining. Images were acquired under a microscope (C-5050, Olympus, Japan). The results were independently evaluated in a blinded manner by two pathologists. The intensity of $\mathrm{BDH} 2$ staining was scored as described. ${ }^{20}$

Cell proliferation assay

The cell proliferative capacity was measured by MTT assay as previously described. ${ }^{20}$

Acetoacetate colorimetric detection assay

Acetoacetate Colorimetric Assay Kit (Biovision, Milpitas, CA, USA) was used to determine intracellular acetoacetate content, following the manufacturer's instructions.
Colony-formation assay

Cells were seeded in six-well plates at $2 \times 10^{2}$ cells per well. After 14 days, Giemsa-stained colonies formed were photographed and counted by using Quantity One v4.4.0 (Bio-Rad, USA).

In vivo tumorigenicity assay

Five 5-week-old male BALB/c-nu nude mice (Vital River Laboratory Animal Technology, China) were injected with $1.0 \times 10^{6}$ pCMV6Entry-5-8F cells and an equal number of $\mathrm{BDH} 2-5-8 \mathrm{~F}$ cells into the left flank by subcutaneous injection. All mice were raised by professional breeders from Laboratory Animal Center of Guangxi Medical University in a barrier system with certain temperature and humidity in the SPF animal lab and were randomly divided into two groups. The tumour volume was assessed by 2D measurements at $0,3,5,8,11$ and 14 days. Tumour volume was calculated as volume $\left(\mathrm{mm}^{3}\right)=$ length $\times$ width ${ }^{2} \times 0.5$. Then mice were killed by cervical dislocation and tumours were removed. All animal experimental procedures were performed under protocol No. 201808022, approved and regulated by The Animal Care \& Welfare Committee of Guangxi Medical University. All methods were carried out in accordance with the Guiding Opinions on the Treatment of Laboratory Animals issued by the Ministry of Science 
a

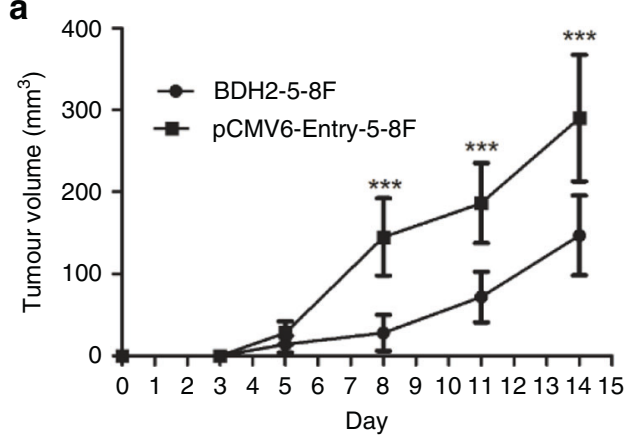

b

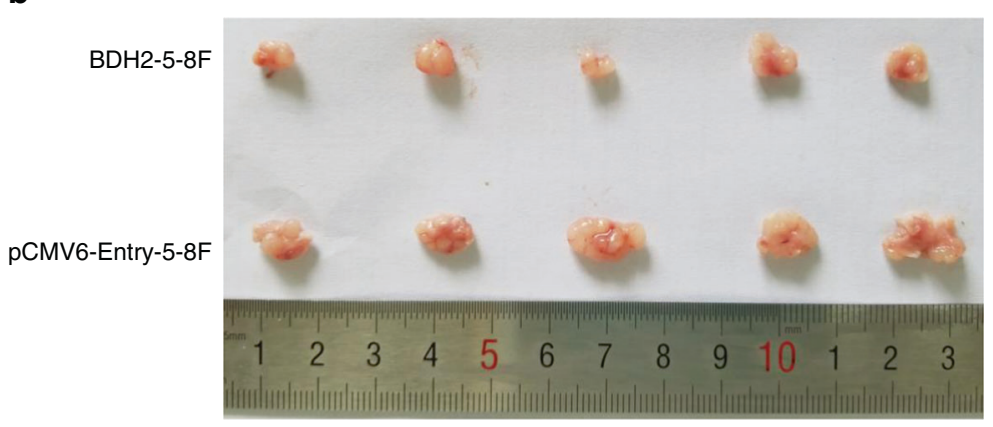

C

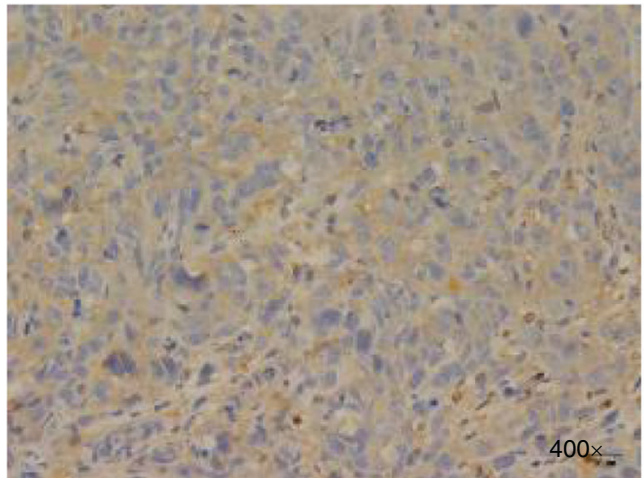

$\mathrm{BDH} 2-5-8 \mathrm{~F}$

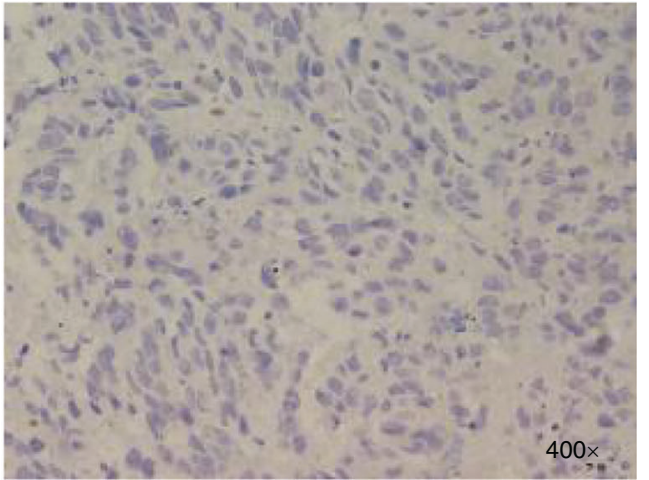

pCMV6-Entry-5-8F

Fig. 3 BDH2 suppresses NPC tumour growth in vivo. a The volume of tumours derived from BDH2-5-8F-1 and pCMV6-5-8F-1 cell injection in mice was measured at days $0,3,5,8,11$ and 14 after inoculation. b Xenograft tumours were removed at 14 days after inoculation in nude mice $(n=5)$. Data are mean \pm SD. $c$ Immunohistochemical staining of BDH2 in removed tumours (magnification $\times 400$ ). ${ }^{* * *} P<0.001$

and Technology of the People's Republic of China and the Laboratory Animal-Guideline for Ethical Review of Animal Welfare issued by the National Standard GB/T35892-2018 of the People's Republic of China.

cDNA microarray analysis and bioinformatics analysis Affymetrix GeneChip Genome U133 Plus 2.0 expression array and Gene-Cloud of Biotechnology Information (GCBI) platform (www. gcbi.com.cn) were used to analyse genes with differential expression between $\mathrm{BDH} 2-5-8 \mathrm{~F}$ and pCMV6-Entry-5-8F cells. The microarray data are available via the following accession identifier on the NCBI-GEO database: GSE119642. The expression level of BDH2 in TCGA data was performed by using GEPIA (http:// gepia.cancer-pku.cn).

Wound-healing assay

Cells $\left(8 \times 10^{5}\right.$ per well) were seeded into 12 -well plates and allowed to adhere overnight with DMEM medium without serum. Monolayer cells were scratched by using the ibidi Culture-Insert (No. 80209, ibidi, Germany). Images were acquired under a light microscope (TS100, Nikon, Japan) two times (0 and 18h), and analysed by using Image J v1.51k (NIH, USA).

Transwell assay

Cells $\left(2.5 \times 10^{4}\right.$ per well) were seeded in the upper chamber of 24well Bio-Coat Invasion Chambers (BD, USA) coated with Matrigel. The lower chamber was filled with DMEM medium with 10\% FBS. Non-invading cells were removed by using a cotton-tipped swab at $48 \mathrm{~h}$. Migratory and invasive cells on the lower membrane surface were fixed with $1 \%$ paraformaldehyde, stained with $0.5 \%$ crystal violet and photographed.
Western-blot analysis

Equal amounts of protein were separated by electrophoresis on 4-12\% SDS-PAGE and transferred to the nitrocellulose membrane (Thermo Fisher Scientific, USA), which was blocked in $5 \%$ bovine serum albumin (BSA) in $1 \times$ Tris-buffered saline containing $0.1 \%$ Tween 20 . The membrane was incubated with primary antibodies at $4{ }^{\circ} \mathrm{C}$ overnight followed by the appropriate peroxidaseconjugated secondary antibodies at room temperature for $2 \mathrm{~h}$. LI-COR Odyssey (Lincoln, NE, USA) was used to detect fluorescent signals.

Iron colorimetric detection assay

Iron Colorimetric Assay Kit (Biovision, Milpitas, USA) was used to determine intracellular iron content, following the manufacturer's instructions.

Statistical analysis

All data were analysed by using SPSS 17.0 (SPSS Inc., Chicago, IL, USA). Data are expressed as mean \pm SD. The statistical analysis was performed by Independent-Sample Test. Statistical significance was considered at $p<0.05$.

\section{RESULTS}

$\mathrm{BDH} 2$ is downregulated in NPC cell lines and tissue

To reveal the potential role for $\mathrm{BDH} 2$ in NPC, we examined the mRNA expression of $\mathrm{BDH} 2$ in NPC primary tumours and NPC cell lines by real-time RT-PCR. BDH2 level was decreased in six NPC cell lines (HONE1, HK1, CNE1, CNE2, TW03 and 5-8F) as compared with two noncancerous nasopharyngeal epithelial cell lines (NP69 and NP460) (Fig. 1a). As well, BDH2 expression was lower in NPC 
tissues than NNE tissues (Fig. 1b). We also evaluated BDH2 expression in patient-derived biopsies by immunohistochemistry staining (IHC). BDH2 protein was detected in the cytoplasm of both NNE and NPC cells. Notably, BDH2 expression was significantly lower in NPC than NNE (Fig. 1C).

To confirm our findings, we analysed RNA-sequencing data from the cancer genome atlas (TCGA) and found BDH2 transcription downregulated in several kinds of cancer, including cervical and endocervical cancers (CESC), cholangiocarcinoma (CHOL), prostate adenocarcinoma (PRAD) and lung squamous cell carcinoma (LUSC) (Supplementary Fig. 1), suggesting that inactivation of $\mathrm{BDH} 2$ may be a common event in human cancers.

Ectopic expression of $\mathrm{BDH} 2$ inhibits the proliferation and colony formation of NPC cells

To access the function of $\mathrm{BDH} 2, \mathrm{NPC}$ cell line $5-8 \mathrm{~F}$ was stably transfected with control vector and $\mathrm{BDH} 2$ expression plasmid, respectively. We had three single clones of pCMV6-Entry-5-8F ( $p \_1, p \_2$ and $p_{-} 3$ ) and $\mathrm{BDH} 2-5-8 \mathrm{~F}$ (b_1, b_3 and b_6), respectively. The expression of $\mathrm{BDH} 2$ was confirmed by both real-time RT-PCR and western-blot assay (Fig. 2a, b). To further confirm the function of $\mathrm{BDH} 2$, we detected an increase in intracellular acetoacetate level in $\mathrm{BDH} 2-5-8 \mathrm{~F}$ cells (Fig. 2c). In addition, $\mathrm{BDH} 2-5-8 \mathrm{~F}$ cells grew more slowly than control cells (Fig. 2d), with reduced capacity for colony formation (Fig. 2e).
$\mathrm{BDH} 2$ suppresses NPC tumour growth in vivo

To determine whether the effect of $\mathrm{BDH} 2$ expression on tumour suppression could be reproduced in vivo, we injected $\mathrm{BDH} 2-5-8 \mathrm{~F}$ and pCMV6-Entry-5-8F cells in the left flank of nude mice. Fourteen days later, mice were killed, and tumour volume was smaller with inoculation of $\mathrm{BDH} 2-5-8 \mathrm{~F}$ than pCMV6-Entry-5-8F cells (Fig. 3a, b). IHC of tumours confirmed the increased expression of $\mathrm{BDH} 2$ in tumours from $\mathrm{BDH} 2-5-8 \mathrm{~F}$ cell lines (Fig. $3 \mathrm{C}$ ).

$\mathrm{BDH} 2$ reverses the epithelial-mesenchymal transition (EMT) in NPC cells

By microarray analysis, we found that the top two genes with differential expression, EPCAM ( 5-fold) and SPARC ( 18-fold), involved in EMT, were altered by overexpressing $\mathrm{BDH} 2$ in $5-8 \mathrm{~F}$ cells (Supplementary Table 1). Therefore, we analysed 21 EMT markers (Fig. 4a). The typical EMT marker, $\mathrm{CDH} 1$ (upregulated 3fold) was influenced by $\mathrm{BDH} 2$ expression. As well, the transcription levels of EPCAM and $\mathrm{CDH} 1$ were upregulated in $\mathrm{BDH} 2-5-8 \mathrm{~F}$ versus PCMV6-Entry-5-8F cells, and those of SPARC, VIM and MMP2 were downregulated (Fig. 4b). In addition, 5-8F cells with overexpressed $\mathrm{BDH} 2$ showed significantly increased protein expression of E-cadherin, and reduced expression of $\beta$-catenin, SPARC and VIM (Fig. 5C), while the expression of EPCAM was not affected by $\mathrm{BDH} 2$. These data suggest that the expression level of $\mathrm{BDH} 2$ might contribute to the migration and invasion of NPC cells.

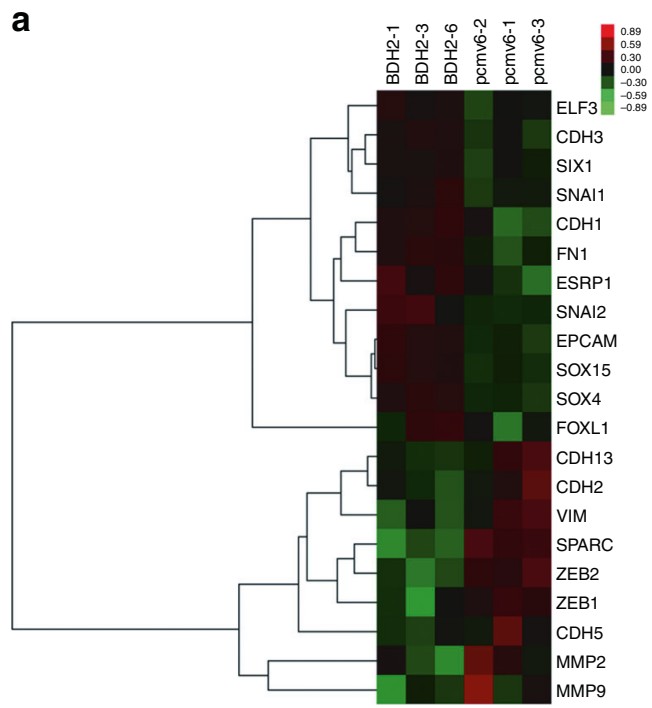

b
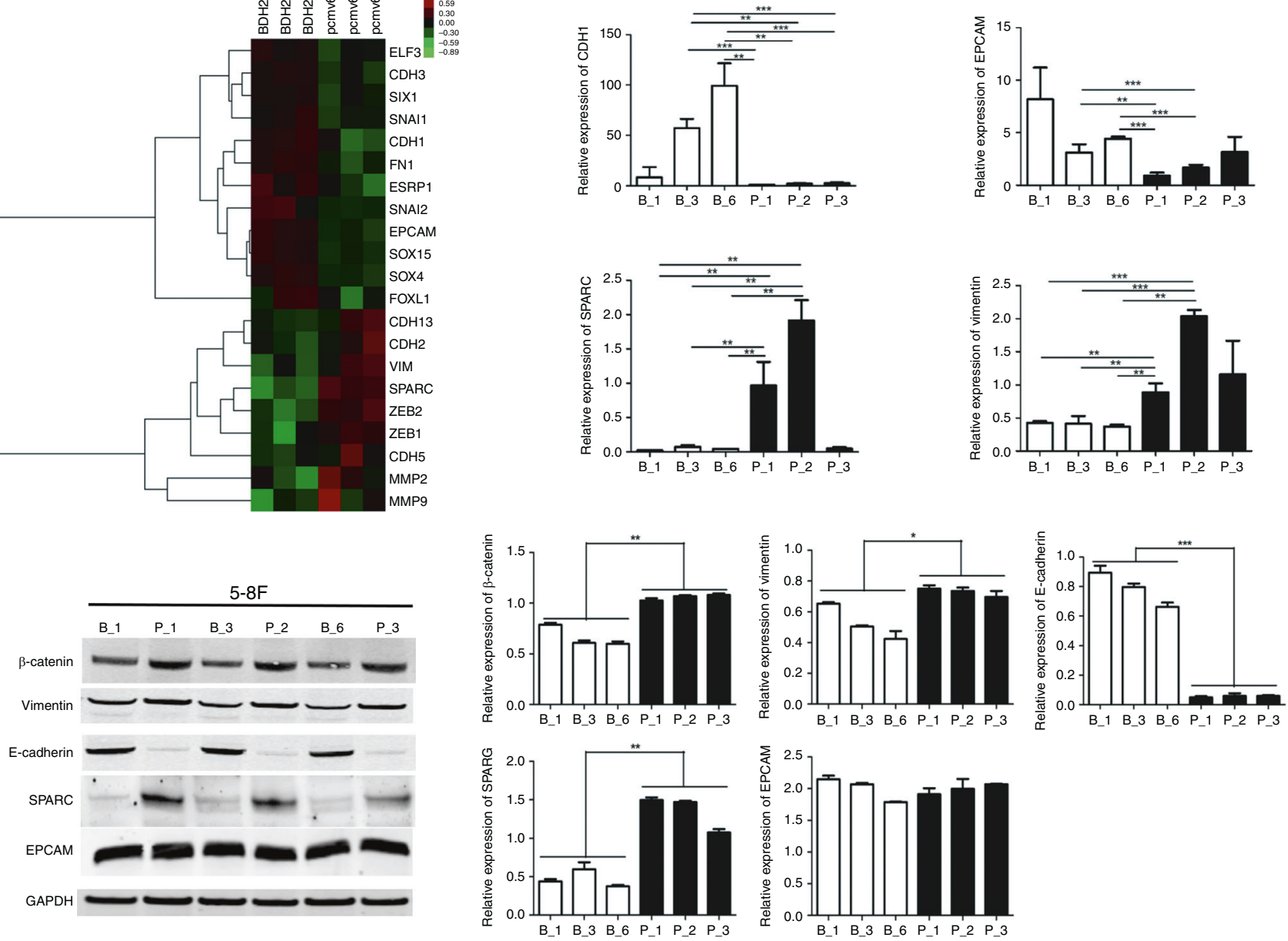

Fig. 4 BDH2 reverses the epithelial-mesenchymal transition (EMT) in NPC cells. a Microarray assay: heatmap showing expression of 21 genes involved in EMT in BDH2-overexpressing 5-8F cells as compared with pCMV6-Entry-5-8F cells. b Relative expression of CDH1, EPCAM, SPARC and VIM analysed by real-time RT-PCR in BDH2-5-8F and pCMV6-Entry-5-8F cells. c Western-blot analysis of protein expression of E-cadherin, $\beta$-catenin, vimentin, SPARC and EPCAM in cells. GAPDH was used as an endogenous control. Data are mean \pm SD $(n=3)$. ${ }^{*} P<0.05$; ${ }^{* *} P<0.01$; $* * * P<0.001$ 


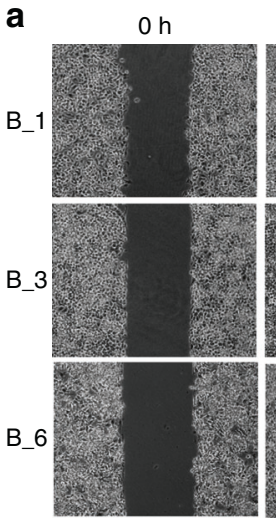

$18 \mathrm{~h}$

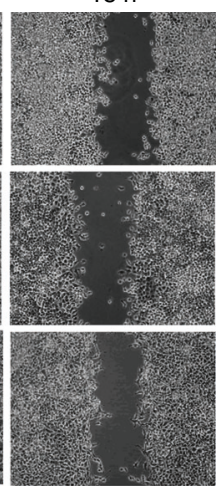

$\mathrm{Oh}$

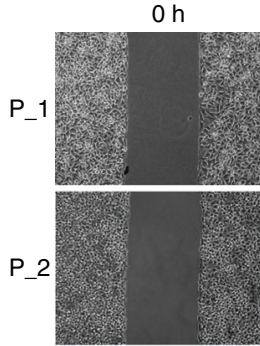

P_3

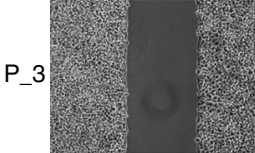

$18 \mathrm{~h}$

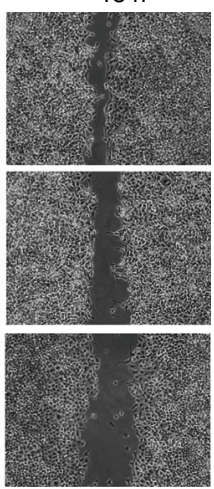

b
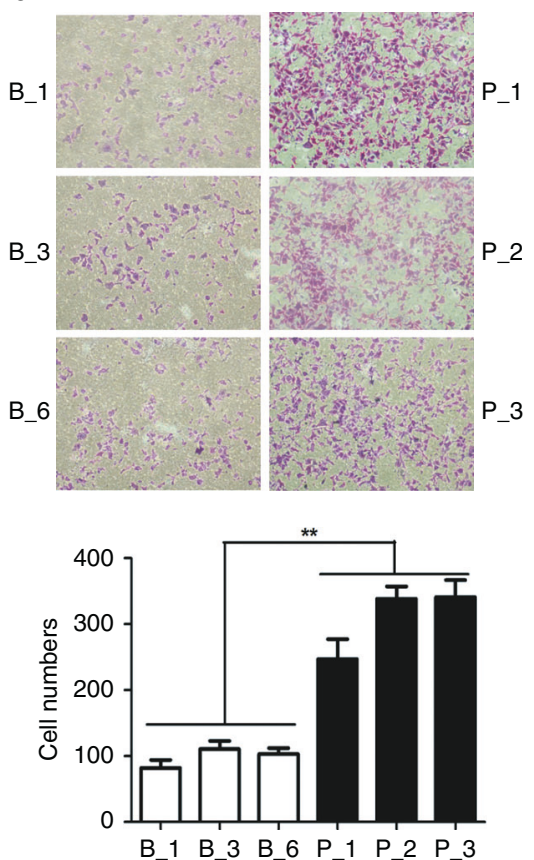

Fig. 5 Ectopic expression of $\mathrm{BDH} 2$ inhibits migration and invasion of NPC cells. a Migration of three sub-colonies of $\mathrm{BDH} 2-5-8 \mathrm{~F}$ and pCMV6-5-8F cells examined by wound-healing assay at 0 and $18 \mathrm{~h}$. The percentage of wound width for each sample was calculated. Magnification $\times 100$. b Transwell assay of invasive capacity of $\mathrm{BDH} 2-5-8 \mathrm{~F}$ and $\mathrm{pCMV6}-5-8 \mathrm{~F}$ cells. The blue dots represent the invading cells stained with crystal violet. The number of invading cells was counted. Data are mean \pm SD $(n=3)$. ${ }^{*} P<0.05,{ }^{* *} P<0.01$

Ectopic expression of $\mathrm{BDH} 2$ inhibits migration and invasion of NPC cells

To investigate the effect of $\mathrm{BDH} 2$ on the motility and invasive capacities of NPC cells, we performed wound-healing assay and Transwell assay, respectively. We observed that the gap closure is slower in $\mathrm{BDH} 2-5-8 \mathrm{~F}$ cells (Fig. $5 \mathrm{a}$ ), and fewer $\mathrm{BDH} 2-5-8 \mathrm{~F}$ cells invaded the extracellular matrix gel in contrast with control cells (Fig. 5b), suggesting that higher expression of $\mathrm{BDH} 2$ attenuates the metastasis of NPC cells by reversing EMT.

$\mathrm{BDH} 2$ inhibits proliferation by reducing intracellular iron content $\mathrm{BDH} 2$ deficiency has been shown to promote iron overload. ${ }^{21}$ To confirm the ability of $\mathrm{BDH} 2$ to regulate iron levels in NPC cells, we detected intracellular iron deposits by iron colorimetric assay. The iron level was lower with two single clones of $\mathrm{BDH} 2-5-8 \mathrm{~F}$ cells than two clones of pCMV6-Entry-5-8F cells (Fig. 6a), which suggests that downregulation of $\mathrm{BDH} 2$ causes iron retention in NPC cells. Re-expression of $\mathrm{BDH} 2$ may decrease intracellular iron content, thereby inhibiting the proliferation of NPC cells.

Elevated iron amount increases proliferation, migration and invasion of NPC cells

To further evaluate the effect of iron on growth, we treated 5-8F and HK1 cells with different concentrations of deferasirox, an iron inhibitor. Deferasirox decreased the proliferation of these two cell lines; the inhibition effect was more obvious with increased deferasirox concentration (Fig. 6b; Supplementary Fig. 1A). With iron supplemented in increasing concentrations, the capacity for cell proliferation was restored gradually (Fig. 6c; Supplementary Fig. 2B). Thus, intracellular iron level may be positively associated with the growth ability of NPC cells. High intracellular iron level has been verified to contribute to metastasis progression. ${ }^{22}$ EGFR also plays a role in regulating iron homoeostasis to increase iron import for cancer cell. ${ }^{23}$ The wound-healing assay and Transwell assay were performed to determine the effect of the iron supplement on the motility and invasive capacities of NPC cells.
The data showed that iron supplement significantly increased the migration of $5-8 \mathrm{~F}$ cells at concentration $2 \mu \mathrm{M}$ (Fig. $6 \mathrm{~d}$ ). In addition, 0.5 and $1 \mu \mathrm{M}$ iron supplement significantly promoted invasion (Fig. 6e). We demonstrated that iron supplement increases migration and invasion of NPC cells. Moreover, as shown in Supplementary Fig. 3, the migration of HK1 cells transiently expressed $\mathrm{BDH} 2$ faster after iron supplementation. This evidence supports that the motility of NPC cells impeded by overexpressing $\mathrm{BDH} 2$ relies on the iron decline.

\section{DISCUSSION}

Here, we report for the first time that $\mathrm{BDH} 2$ is inactivated in NPC. We also found that the transcription of $\mathrm{BDH} 2$ was downregulated in a variety of tumours, indicating that the lower expression of $\mathrm{BDH} 2$ is universal in malignancies. To date, what causes $\mathrm{BDH} 2$ inactivation in tumour has not been addressed yet. It has been reported that $\mathrm{BDH} 2$ expression is downregulated during inflammatory and ER stress response in macrophages. ${ }^{24}$ One could speculate that the inflammatory microenvironment of tumour cells could negatively influence $\mathrm{BDH} 2$ expression activity. In contrast, the expression of $\mathrm{BDH} 2$ was reported to be aberrantly high in patients with acute myeloid leukaemia and oesophageal carcinoma, $^{12}$ which imply a tumour-specific effect of $\mathrm{BDH} 2$ expression.

In this study, we have investigated the role of $\mathrm{BDH} 2$ in the pathogenesis of NPC. Ectopic expression of BDH2 in NPC cells reduced cell proliferation, colony formation and suppressed tumorigenesis in the in vivo model. The restored expression of $\mathrm{BDH} 2$ suppressed migration and invasion of NPC cells by reversing the EMT process. Altogether, our data support a role of $\mathrm{BDH} 2$ as a tumour-suppressor gene in NPC.

Proper energy metabolism is an essential factor in the survival and progression of normal cells through a cell cycle. Metabolic alterations have been observed in various malignancies and considered as a hallmark of cancer. ${ }^{25}$ Metabolic reprogramming of 
cancer cells provides additional energy to support their malignant behaviour, characterised by accelerated proliferation, resistance to apoptosis, evasion of immune surveillance and maintenance of the status of cancer stem cells. ${ }^{26-28}$ Previously, we reported that accumulating lipid droplets (LDs) in NPC cells is positively correlated with their malignant behaviour, indicating that LDs might be an energy fuel of NPC. ${ }^{29}$ In several types of cancer, obesity and excess accumulation of adipose tissues are widely considered as high-risk factors. ${ }^{30}$ In addition, lipid accumulation significantly reduces the drug efficacy of chemotherapy and antiangiogenic drug in cancer. ${ }^{31,32}$ Targeting lipid metabolism combined with other treatment may be a novel strategy for cancer patients with multidrug resistance or obesity.

In organs other than the liver, $\mathrm{BDH} 2$ dehydrates $\beta$-hydroxybutyric acid to form one of the endogenous ketone body molecules, acetoacetate, ${ }^{9}$ considered to be a nutritional source for tumours carrying the V600E mutant form of BRAF protooncogene. ${ }^{3,34}$ Interestingly, ketone bodies also reduce the growth of pancreatic cancer and cause apoptosis. ${ }^{35}$ We found that restoring the expression of $\mathrm{BDH} 2$ in NPC cells increased the intracellular acetoacetate level. Early, we showed that the use of exogenous acetoacetate does not affect the migration of NPC cells, ${ }^{20}$ which suggests that the tumour-suppressor function of $\mathrm{BDH} 2$ still does not rely on acetoacetate production. Intriguingly, a recent study found that acetoacetate produced by overexpressing
$\mathrm{BDH} 2$ in liver cancer cells inactivates the macrophage migratory inhibitory factor (MIF), therefore impeding the recruitment of tumour-associated macrophage (TAM), an important target for cancer treatment. ${ }^{36,37}$ We speculated that acetoacetate affects cancer cells through the paracrine mode of action, other than autocrine. More experiments are necessary to address this interesting issue in the future.

Iron metabolism is a chain of chemical reactions that supports iron homoeostasis at both the systemic and cellular levels. Iron is directly related to cell proliferation and growth. It can generate reactive oxygen species (ROS) by participating in the Fenton reaction that produces hydroxyl radicals. ROS can damage DNA and be mutagenic. Thus, iron is fundamental to cell survival and may also be associated with carcinogenesis. ${ }^{38}$

In recent years, numerous disorders, such as cancer and neurodegenerative diseases, have been linked to deregulated iron homoeostasis. ${ }^{33,34}$ Cellular iron metabolism of iron includes three major processes: iron uptake, storage and export of iron. Iron carrier proteins, such as transferrin receptors in glioblastoma and ferritin in serum, were upregulated, thereby increasing iron uptake. ${ }^{35,36}$ The decrease in expression of an iron exporter protein ferroportin is associated with a poor prognosis for breast cancer. ${ }^{39}$ In addition, accumulation of iron supports cancer stem cells and induces drug resistance, transforming the cells into a malignant phenotype and leading to treatment failure. ${ }^{35,37}$ In patients with a

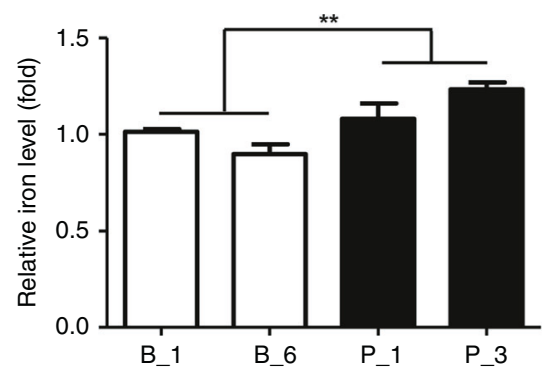

d
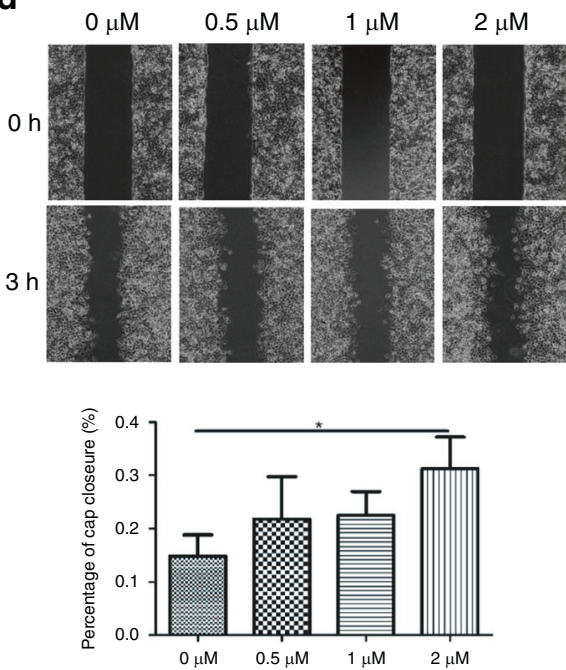

b

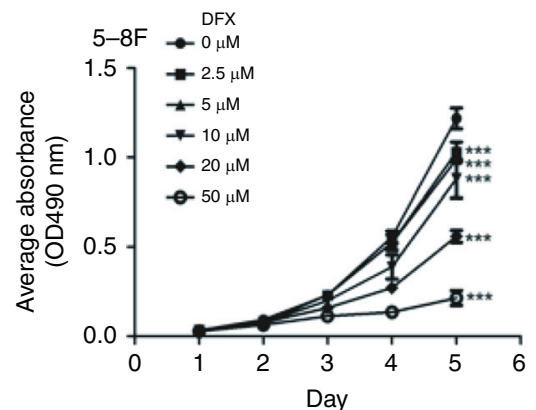

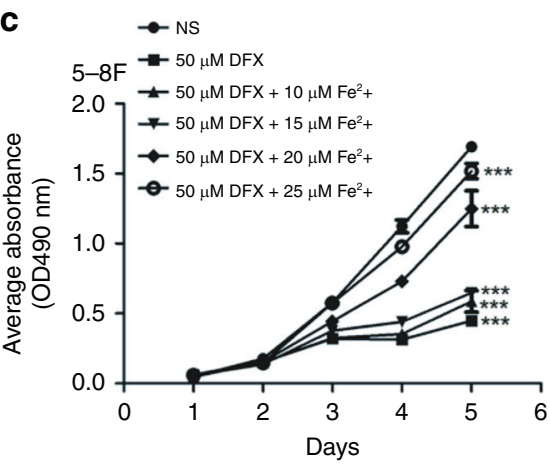

e
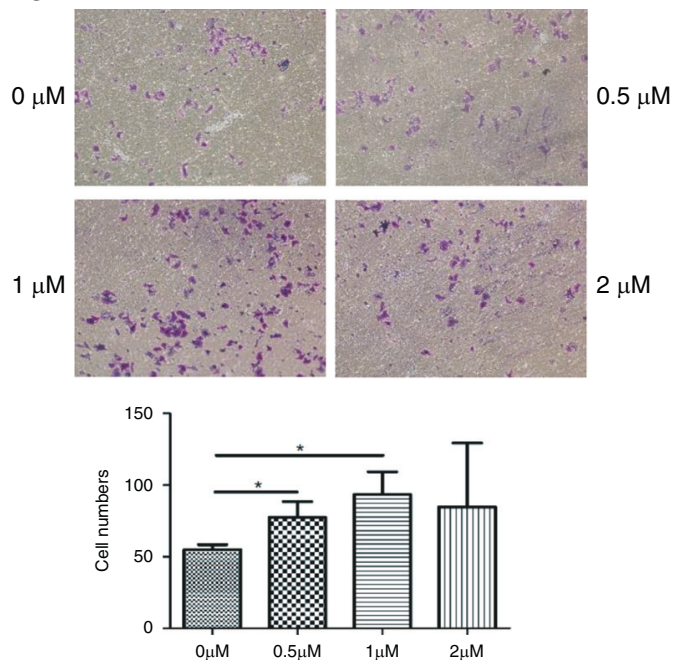

Fig. 6 Iron retention accelerates cell proliferation, migration and invasion of NPC cells. a Relative concentration of intracellular iron calculated as the total amount of intracellular iron per microgram protein in two sub-colonies each of BDH2-5-8F and pCMV6-Entry-5-8F cells. b MTT assay of proliferation of 5-8 F cells under deferasirox (DFX) treatment. c Proliferation of cells after adding iron (II) sulfate heptahydrate to saturate the iron-chelating ability of deferasirox $(50 \mu \mathrm{M})$. Data are mean \pm SD $(n=5)$. d Wound-healing assay was performed in $5-8 \mathrm{~F}$ cells treated with iron (II) sulfate heptahydrate at $0,0.5,1$ and $2 \mu \mathrm{M}$, respectively. The wound width was measured at 0 and $3 \mathrm{~h}$. Magnification $\times 100$. e Invasive capacity of 5-8F cells, with iron supplement, was assessed by Transwell assay. The number of invading cells was counted. Data are mean \pm SD $(n=3) .{ }^{*} P<0.05 ;{ }^{* *} P<0.01 ; * * * P<0.001$ 
NPC, higher serum ferritin levels are closely associated with the development of metastasis. ${ }^{40}$ Conversely, high expression of the ferritin heavy chain in NPC cell lines significantly reduces the ability of these cells to proliferate. ${ }^{41}$ Iron metabolism is not fully understood in NPC yet.

$\mathrm{BDH} 2$ mediates the formation of 2,5-dihydroxybenzoic acid (2,5DHBA), a siderophore. Siderophores are small high-affinity ironchelating compounds that function in transporting iron across cell membranes. $\mathrm{BDH} 2$ plays a crucial role in intracellular homoeostasis of iron and regulates the immune function of macrophages. $^{42,43}$ Siderophore transports ferrous iron to mitochondria, since its absence leads to iron deficiency in mitochondria, and as a result, to an increase in the level of iron in cells. ${ }^{10}$ Mitochondria are the main place of iron utilisation. The imported iron is used to synthesise iron-sulfur clusters, which are important for the activity of the citric acid cycle enzymes, oxidative phosphorylation and mitochondrial respiration. ${ }^{44}$ The lack of siderophore contributes to the abnormal accumulation of intracellular iron and increases cell proliferation. ${ }^{10}$ Our results indicate that inactivation of $\mathrm{BDH} 2$ leads to iron retention in nasopharyngeal epithelial cells, so it may be important for carcinogenesis and development of NPC.

Approaches targeting cellular iron levels and iron-mediated signalling to inhibit tumour growth have been developed and applied in cancer therapy. The use of iron chelators can cause cell cycle arrest, which will subsequently lead to apoptosis, which suggests the use of iron chelators as potential anticancer agents. ${ }^{45-48}$ Artemisinin is toxic only to cancer cells that contain more iron and kills cancer cells by increasing the level of ROS. ${ }^{49}$ ROS are required to cause DNA damage during radiotherapy, the main strategy for NPC treatment. Our study showed for the first time that an iron chelator, desferrioxamine, suppresses the proliferation of NPC cells in vitro, so iron chelators can be useful for treating NPCs by suppressing cell growth and proliferation or participating in redox reactions as a radiation therapy enhancer. Thus, iron metabolism management may be a new therapeutic target in the treatment of NPC.

Thus, we found that BDH2 expression is downregulated in NPC. Overexpression of $\mathrm{BDH} 2$ in NPC cells decreased the levels of intracellular iron, thereby inhibiting cell proliferation. In addition, overexpression of $\mathrm{BDH} 2$ reduced invasion and migration of NPC cells by reversing EMT. Our results revealed a new mechanism for the carcinogenesis of NPC in that iron metabolism may influence the development of NPC. Thus, iron metabolism management can be a potential approach to prevention and treatment in NPC.

\section{ACKNOWLEDGEMENTS}

We thank Dr. Liudmila Matskova (Institute of Living Systems, Immanuel Kant Baltic Federal University, Kaliningrad, Russia) for his useful comment.

\section{AUTHOR CONTRIBUTIONS}

Z.Z., R.S.W. and X.Y.Z. were involved in the design of the project. B.L., Z.P.L., Y.X.M., C.P.D., S.H.Z. and Y.S.L. were involved in performing experiments and collecting data. W.L.Z., X.L.X., W.M.C., F.G.F. and X.H.Z. were involved in analysis and interpretation of data. X.X., P.L. and G.W.H. were involved in the acquisition of clinical samples. All authors have contributed to the writing of the paper and have critically reviewed it.

\section{ADDITIONAL INFORMATION}

Supplementary information is available for this paper at https://doi.org/10.1038/ s41416-019-0638-8.

Competing interests: The authors declare no competing interests.

Ethics approval and consent to participate: The study was approved by the Research Ethics Committee of Guangxi Medical University (Nanning, China). Written informed consent was obtained from all patients. All methods were performed in accordance with the relevant guidelines and regulations. Animal experimental procedures were performed under protocol No. 201808022, approved and regulated by The Animal Care \& Welfare Committee of Guangxi Medical University. The study was performed in accordance with the Declaration of Helsinki.

Funding: This work was supported by the National Natural Science Foundation of China $(81772882,81660458)$ and Promotion of Basic Ability of Young and Middleaged Teachers in Universities in Guangxi (KY2016YB080).

Consent to publish: All authors read and consented to publication of the paper.

Data availability: The microarray data have been deposited to the NCBI-GEO database (https://www.ncbi.nlm.nih.gov/geo/) with the data set identifier GSE119642.

Note: This work is published under the standard license to publish agreement. After 12 months the work will become freely available and the license terms will switch to a Creative Commons Attribution 4.0 International (CC BY 4.0).

Publisher's note Springer Nature remains neutral with regard to jurisdictional claims in published maps and institutional affiliations.

\section{REFERENCES}

1. Chang, E. T. \& Adami, H. O. The enigmatic epidemiology of nasopharyngeal carcinoma. Cancer Epidemiol., Biomarkers Prev. 15, 1765-1777 (2006).

2. McDermott, A. L., Dutt, S. N. \& Watkinson, J. C. The aetiology of nasopharyngeal carcinoma. Clin. Otolaryngol. Allied Sci. 26, 82-92 (2001).

3. Tao, Q. \& Chan, A. T. Nasopharyngeal carcinoma: molecular pathogenesis and therapeutic developments. Expert Rev. Mol. Med. 9, 1-24 (2007).

4. Yu, K. H., Leung, S. F., Tung, S. Y., Zee, B., Chua, D. T., Sze, W. M. et al. Survival outcome of patients with nasopharyngeal carcinoma with first local failure: a study by the Hong Kong Nasopharyngeal Carcinoma Study Group. Head \& Neck 27, 397-405 (2005).

5. Legendre, C. \& Garcion, E. Iron metabolism: a double-edged sword in the resistance of glioblastoma to therapies. Trends Endocrinol. Metab. 26, 322-331 (2015).

6. Toyokuni, S. Role of iron in carcinogenesis: cancer as a ferrotoxic disease. Cancer Sci. 100, 9-16 (2009).

7. Pinnix, Z. K., Miller, L. D., Wang, W., D’Agostino, R. Jr., Kute, T., Willingham, M. C. et al. Ferroportin and iron regulation in breast cancer progression and prognosis. Sci. Transl. Med. 2, 43 ra56 (2010).

8. Guo, K., Lukacik, P., Papagrigoriou, E., Meier, M., Lee, W. H., Adamski, J. et al. Characterization of human DHRS6, an orphan short chain dehydrogenase/ reductase enzyme: a novel, cytosolic type 2 R-beta-hydroxybutyrate dehydrogenase. J. Biol. Chem. 281, 10291-10297 (2006).

9. Grabacka, M., Pierzchalska, M., Dean, M. \& Reiss, K. Regulation of ketone body metabolism and the role of PPARalpha. Int. J. Mol. Sci.; 17; https://doi.org/ 10.3390/ijms17122093 (2016).

10. Liu, Z., Velpula, K. K. \& Devireddy, L. 3-Hydroxybutyrate dehydrogenase-2 and ferritin-H synergistically regulate intracellular iron. FEBS J. 281, 2410-2421 (2014).

11. Yang, W. C., Tsai, W. C., Lin, P. M., Yang, M. Y., Liu, Y. C., Chang, C. S. et al. Human $\mathrm{BDH} 2$, an anti-apoptosis factor, is a novel poor prognostic factor for de novo cytogenetically normal acute myeloid leukemia. J. Biomed. Sci. 20, 58 (2013).

12. Zang, W., Wang, T., Wang, Y., Chen, X., Du, Y., Sun, Q. et al. Knockdown of long non-coding RNA TP73-AS1 inhibits cell proliferation and induces apoptosis in esophageal squamous cell carcinoma. Oncotarget 7, 19960-19974 (2016).

13. Maurer, G. D., Brucker, D. P., Bahr, O., Harter, P. N., Hattingen, E., Walenta, S. et al. Differential utilization of ketone bodies by neurons and glioma cell lines: a rationale for ketogenic diet as experimental glioma therapy. BMC Cancer 11, 315 (2011).

14. Li, H. M., Man, C., Jin, Y., Deng, W., Yip, Y. L., Feng, H. C. et al. Molecular and cytogenetic changes involved in the immortalization of nasopharyngeal epithelial cells by telomerase. Int. J. Cancer 119, 1567-1576 (2006).

15. Tsao, S. W., Wang, X., Liu, Y., Cheung, Y. C., Feng, H., Zheng, Z. et al. Establishment of two immortalized nasopharyngeal epithelial cell lines using SV40 large T and HPV16E6/E7 viral oncogenes. Biochimica et Biophysica Acta 1590, 150-158 (2002).

16. Glaser, R., Zhang, H. Y., Yao, K. T., Zhu, H. C., Wang, F. X., Li, G. Y. et al. Two epithelial tumor cell lines (HNE-1 and HONE-1) latently infected with Epstein-Barr virus that were derived from nasopharyngeal carcinomas. Proc. Natl Acad. Sci. USA 86, 9524-9528 (1989).

17. Lin, C. T., Chan, W. Y., Chen, W., Huang, H. M., Wu, H. C., Hsu, M. M. et al. Characterization of seven newly established nasopharyngeal carcinoma cell lines. Lab. Investig. 68, 716-727 (1993).

18. Yao, K. T. [Establishment of a novel cell line derived from nasopharyngeal carcinoma]. Zhonghua zhong liu za zhi [Chinese Journal of Oncology] 12, 34-36 (1990).

19. Yao, K. T., Zhang, H. Y., Zhu, H. C., Wang, F. X., Li, G. Y., Wen, D. S. et al. Establishment and characterization of two epithelial tumor cell lines (HNE-1 and 
HONE-1) latently infected with Epstein-Barr virus and derived from nasopharyngeal carcinomas. Int. J. Cancer 45, 83-89 (1990).

20. Luo, W., Qin, L., Li, B., Liao, Z., Liang, J., Xiao, X. et al. Inactivation of HMGCL promotes proliferation and metastasis of nasopharyngeal carcinoma by suppressing oxidative stress. Sci. Rep. 7, 11954 (2017).

21. Liu, Z., Ciocea, A. \& Devireddy, L. Endogenous siderophore 2,5-dihydroxybenzoic acid deficiency promotes anemia and splenic iron overload in mice. Mol. Cell. Biol. 34, 2533-2546 (2014).

22. Fouani, L., Menezes, S. V., Paulson, M., Richardson, D. R. \& Kovacevic, Z. Metals and metastasis: exploiting the role of metals in cancer metastasis to develop novel anti-metastatic agents. Pharmacol. Res. 115, 275-287 (2017).

23. Menezes, S. V., Sahni, S., Kovacevic, Z. \& Richardson, D. R. Interplay of the ironregulated metastasis suppressor NDRG1 with epidermal growth factor receptor (EGFR) and oncogenic signaling. J. Biol. Che. 292, 12772-12782 (2017).

24. Zughaier, S. M., Stauffer, B. B. \& McCarty, N. A. Inflammation and ER stress downregulate $\mathrm{BDH} 2$ expression and dysregulate intracellular iron in macrophages. J. Immunol. Res. 2014, 140728 (2014).

25. Hanahan, D. \& Weinberg, R. A. Hallmarks of cancer: the next generation. Cell 144, 646-674 (2011)

26. Chang, C. H., Qiu, J., O'Sullivan, D., Buck, M. D., Noguchi, T., Curtis, J. D. et al. Metabolic competition in the tumor microenvironment is a driver of cancer progression. Cell 162, 1229-1241 (2015).

27. Lee, N. \& Kim, D. Cancer metabolism: fueling more than just growth. Molecules and Cells 39, 847-854 (2016).

28. Tarrado-Castellarnau, M., de Atauri, P. \& Cascante, M. Oncogenic regulation of tumor metabolic reprogramming. Oncotarget 7, 62726-62753 (2016).

29. Zhou, X., Wei, J., Chen, F., Xiao, X., Huang, T., He, Q. et al. Epigenetic downregulation of the ISG15-conjugating enzyme UbcH8 impairs lipolysis and correlates with poor prognosis in nasopharyngeal carcinoma. Oncotarget 6, 41077-41091 (2015).

30. Cao, Y. Obesity protects cancer from drugs targeting blood vessels. Cell Metab. 27, 1163-1165 (2018).

31. Malvi, P., Chaube, B., Singh, S. V., Mohammad, N., Pandey, V., Vijayakumar, M. V. et al. Weight control interventions improve therapeutic efficacy of dacarbazine in melanoma by reversing obesity-induced drug resistance. Cancer Metab. 4, 21 (2016).

32. Iwamoto, H., Abe, M., Yang, Y., Cui, D., Seki, T., Nakamura, M. et al. Cancer lipid metabolism confers antiangiogenic drug resistance. Cell Metab. 28, 104-117 e105 (2018).

33. Trousil, S. \& Zheng, B. Addicted to AA (Acetoacetate): a point of convergence between metabolism and BRAF signaling. Mol. Cell 59, 333-334 (2015).

34. Xia, S., Lin, R., Jin, L., Zhao, L., Kang, H. B., Pan, Y. et al. Prevention of dietary-fatfueled ketogenesis attenuates BRAF V600E tumor growth. Cell Metab. 25, 358-373 (2017)
35. Shukla, S. K., Gebregiworgis, T., Purohit, V., Chaika, N. V., Gunda, V., Radhakrishnan, $P$. et al. Metabolic reprogramming induced by ketone bodies diminishes pancreatic cancer cachexia. Cancer Metab. 2, 18 (2014).

36. Yang, T., Wang, Y., Dai, W., Zheng, X., Wang, J., Song, S. et al. Increased B3GALNT2 in hepatocellular carcinoma promotes macrophage recruitment via reducing acetoacetate secretion and elevating MIF activity. J. Hematol. Oncol. 11, 50 (2018).

37. Andersson, P., Yang, Y., Hosaka, K., Zhang, Y., Fischer, C., Braun, H. et al. Molecular mechanisms of IL-33-mediated stromal interactions in cancer metastasis. $\mathrm{JCI}$ insight; 3: https://doi.org/10.1172/jci.insight.122375 (2018).

38. Torti, S. V. \& Torti, F. M. Iron and cancer: more ore to be mined. Nat. Rev. Cancer 13, 342-355 (2013).

39. Alkhateeb, A. A. \& Connor, J. R. The significance of ferritin in cancer: anti-oxidation, inflammation and tumorigenesis. Biochimica et Biophysica Acta 1836, 245-254 (2013).

40. Ho, S., Leung, S. F., Leung, W. T., Tsao, S. Y., Kwan, W. H., Choi, P. et al. Strong association between hyperferritinaemia and metastatic disease in nasopharyngeal carcinoma. Eur. J. Cancer Part B, Oral Oncol. 32b, 242-245 (1996).

41. Feng, Y. P., Liu, Q. C., Zhu, J. F., Xie, F. K. \& Li, L. Development and applications of a nasopharyngeal carcinoma Tet-Off cell line. Oncology Lett. 2, 525-530 (2011)

42. Devireddy, L. R., Hart, D. O., Goetz, D. H. \& Green, M. R. A mammalian siderophore synthesized by an enzyme with a bacterial homolog involved in enterobactin production. Cell 141, 1006-1017 (2010).

43. Zughaier, S. M., Kandler, J. L. \& Shafer, W. M. Neisseria gonorrhoeae modulates iron-limiting innate immune defenses in macrophages. PLOS ONE 9, e87688 (2014).

44. Oexle, H., Gnaiger, E. \& Weiss, G. Iron-dependent changes in cellular energy metabolism: influence on citric acid cycle and oxidative phosphorylation. Biochimica et Biophysica Acta 1413, 99-107 (1999).

45. Saeki, I., Yamamoto, N., Yamasaki, T., Takami, T., Maeda, M., Fujisawa, K. et al. Effects of an oral iron chelator, deferasirox, on advanced hepatocellular carcinoma. World J. Gastroenterol. 22, 8967-8977 (2016).

46. Yang, C., Ma, X., Wang, Z., Zeng, X., Hu, Z., Ye, Z. et al. Curcumin induces apoptosis and protective autophagy in castration-resistant prostate cancer cells through iron chelation. Drug Design, Develop. Ther. 11, 431-439 (2017).

47. Lee, J. C., Chiang, K. C., Feng, T. H., Chen, Y. J., Chuang, S. T., Tsui, K. H. et al. The iron chelator, Dp44mT, effectively inhibits human oral squamous cell carcinoma cell growth in vitro and in vivo. Int. J. Mol. Sci. 17; https://doi.org/10.3390/ ijms17091435 (2016).

48. Harima, H., Kaino, S., Takami, T., Shinoda, S., Matsumoto, T., Fujisawa, K. et al. Deferasirox, a novel oral iron chelator, shows antiproliferative activity against pancreatic cancer in vitro and in vivo. BMC Cancer 16, 702 (2016).

49. Lai, H. C., Singh, N. P. \& Sasaki, T. Development of artemisinin compounds for cancer treatment. Investig. New Drugs 31, 230-246 (2013). 